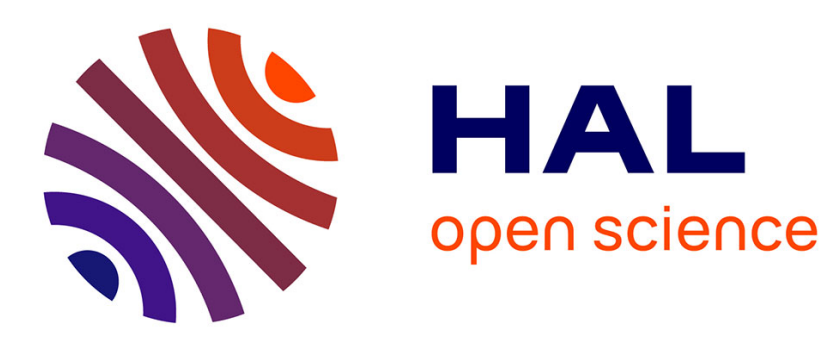

\title{
Boundary data completion: the method of boundary value problem factorization
}

\author{
Amel Ben Abda, Jacques Henry, Fadhel Jday
}

\section{To cite this version:}

Amel Ben Abda, Jacques Henry, Fadhel Jday. Boundary data completion: the method of boundary value problem factorization. Inverse Problems, 2011, 27 (5), 10.1088/0266-5611/27/5/055014 . inria00617511

\section{HAL Id: inria-00617511 \\ https://hal.inria.fr/inria-00617511}

Submitted on 29 Aug 2011

HAL is a multi-disciplinary open access archive for the deposit and dissemination of scientific research documents, whether they are published or not. The documents may come from teaching and research institutions in France or abroad, or from public or private research centers.
L'archive ouverte pluridisciplinaire HAL, est destinée au dépôt et à la diffusion de documents scientifiques de niveau recherche, publiés ou non, émanant des établissements d'enseignement et de recherche français ou étrangers, des laboratoires publics ou privés. 


\title{
Boundary data Completion: The method of boundary value problem factorization
}

\author{
Amel Ben Abda ${ }^{1}$, Jacques Henry ${ }^{2}$ and Fadhel Jday ${ }^{1}$ \\ ${ }^{1}$ LAMSIN ENIT, Campus universitaire de Tunis Tunisie \\ ${ }^{2}$ INRIA-Bordeaux Sud-ouest and IMB, université Bordeaux1, 351, cours de la \\ libération 33405 TALENCE cedex FRANCE \\ E-mail: amel.benabda@enit.rnu.tn, jacques.henry@inria.fr and \\ fadheldj@yahoo.com
}

\begin{abstract}
We consider the following data completion problem for the Laplace equation in the cylindrical domain: $\Omega=] 0, a\left[\times \mathcal{O}, \mathcal{O} \subset \mathbb{R}^{n-1}\right.$ ( $\mathcal{O}$ is a smooth bounded open set and $a>0)$, limited by the faces $\Gamma_{0}=\{0\} \times \mathcal{O}$ and $\Gamma_{a}=\{a\} \times \mathcal{O}$. The Neumann and Dirichlet boundary conditions are given on $\Gamma_{0}$ while no condition is given on $\Gamma_{a}$. The completion data problem consists in recovering a boundary condition on $\Gamma_{a}$. This problem has been known since Hadamard [12] to be ill-posed. The problem is set as an optimal control problem with a regularized cost function. To obtain directly an approximation of the missing data on $\Gamma_{a}$ we use the method of factorization of elliptic boundary value problems. This method allows to factorize a boundary value problem in the product of two parabolic problems. Here it is applied to the optimality system (i.e. jointly on the state and adjoint state equations).
\end{abstract}




\section{Introduction}

In this work we are concerned by solving the Cauchy problem with data given on a part of the boundary in the framework of Laplace's equation. This problem is known to be ill-posed since Hadamard [12]. There are many approaches for solving such an inverse problem, the more popular ones being those of optimal control techniques [10], [20] or approximate control technique [4] or analytic approximation of functions [18]. The quasi-reversibily method was introduced by Lattès and Lions [17] in the late 60's and it allows to regularize a large class of ill-posed problems. After [15], in recent papers [6], [7], [8], [9], L. Bourgeois revisited the method in the framework of Cauchy's problem for Laplace's equation and studied its stability. In this method the ill-posed secondorder PDE problem is replaced by a fourth-order well posed problem depending on a small regularizing parameter. Notice that in almost all these studies the authors use some regularizing techniques. For example we would like to point out the paper [21] where the authors explored a discretized Tikhonov regularization for a Cauchy problem corresponding to an elliptic PDE by reproducing kernel Hilbert space.

In this paper, we consider the following problem:

$$
\left(\mathcal{P}_{0}\right)\left\{\begin{array}{rlc}
\Delta u=0 & \text { in } & \Omega, \\
u=0 & \text { on } & \Sigma, \\
u=T & \text { on } & \Gamma_{0}, \\
\frac{\partial u}{\partial n}=\Phi & \text { on } & \Gamma_{0},
\end{array}\right.
$$

where $\Omega$ is a cylinder in $\left.\mathbb{R}^{n}, \Omega=\right] 0, a\left[\times \mathcal{O}, \mathcal{O} \subset \mathbb{R}^{n-1}\right.$ is a bounded domain, a being a strictly positive real number. Let $\Sigma=] 0, a[\times \partial \mathcal{O}$ be the lateral boundary of $\Omega$ and $\Gamma_{0}=\{0\} \times \mathcal{O}$ and $\Gamma_{a}=\{a\} \times \mathcal{O}$ be the faces. To stress the particular role of the coordinate along the axis of the cylinder we denote it by $x$ while $y \in \mathbb{R}^{n-1}$ denotes the coordinates in the section, perpendicular to the axis. This cylindrical geometry and the Laplace operator make the presentation of the factorization method easy, but the method can be generalized to regular non cylindrical domains and to more general self adjoint second order elliptic operators.

On $\Gamma_{0}$ one has the Cauchy data $(\Phi, T) \in\left(H_{00}^{\frac{1}{2}}(\mathcal{O})\right)^{\prime} \times H_{00}^{\frac{1}{2}}(\mathcal{O}) \ddagger$ whereas no boundary condition is available on $\Gamma_{a}$. The aim of this paper is the recovering of these missing boundary data exploiting the over specified one on the side $\Gamma_{0}$. The existence of a solution of this problem is not insured for arbitrary Cauchy data $(\Phi, T)$. This problem is treated in [1], [11] in a general domain $\Omega$ having a boundary : $\partial \Omega=\Gamma_{0} \cup \Gamma_{1}$. Andrieux et al. [1] rephrase the missing boundary data recovery problem as a control one with two states, each one satisfying one boundary condition on $\Gamma_{0}$, the control being the overdetermined boundary conditions on $\Gamma_{a}$. This method is an iterative one and therefore requires a resolution of the forward problems for each new data $(\phi, T)$. In this work we use the same formulation as in [1] in terms of optimal control but we add

$\ddagger$ Let us recall that the Sobolev space $H_{00}^{\frac{1}{2}}(\mathcal{O})$ is defined in Theorem 11.7, p 72 of [19] as the $\frac{1}{2}$ interpolated space of $H_{0}^{1}(\mathcal{O})$ and $L^{2}(\mathcal{O})$. 
a regularization term to insure the existence and uniqueness of the optimality system [20]. We make use of the factorization method which transforms the elliptic boundary value problem into two parabolic ones. It allows the direct evaluation of the missing boundary data for all Cauchy data $(\Phi, T)$.

\section{The boundary data recovering as an optimal control problem}

If $\varphi, t$ on $\Gamma_{a}$ match the boundary data we will have an overdetermined boundary problem given by:

$$
\left\{\begin{array}{llc}
\Delta u=0 & \text { in } & \Omega, \\
u=0 & \text { on } & \Sigma, \\
\frac{\partial u}{\partial n}=\Phi, \quad u=T & \text { on } & \Gamma_{0} \\
u=t, \quad \frac{\partial u}{\partial n}=\varphi & \text { on } & \Gamma_{a} .
\end{array}\right.
$$

We consider now, the following optimal control problem:

for all $(\eta, \tau) \in\left(H_{00}^{\frac{1}{2}}(\mathcal{O})\right)^{\prime} \times H_{00}^{\frac{1}{2}}(\mathcal{O})$, the state $\left(u_{1}, u_{2}\right)$ is defined by :

$$
\begin{aligned}
& \begin{cases}\Delta u_{1}=0 & \text { in } \Omega, \\
u_{1}=0 & \text { on } \Sigma, \\
u_{1}=T & \text { on } \Gamma_{0}, \quad \frac{\partial u_{1}}{\partial n}=\eta \quad \text { on } \Gamma_{a}\end{cases} \\
& \begin{cases}\Delta u_{2}=0 & \text { in } \Omega, \\
u_{2}=0 & \text { on } \Sigma, \\
\frac{\partial u_{2}}{\partial n}=\Phi & \text { on } \Gamma_{0}, \quad u_{2}=\tau \quad \text { on } \Gamma_{a} .\end{cases}
\end{aligned}
$$

If $(\phi, T)$ are compatible then $u_{1}=u_{2}$ when $(\eta, \tau)=(\varphi, t)$. We introduce the cost function $E(\eta, \tau)$ defined by:

$$
E(\eta, \tau)=\int_{\Omega}\left(\nabla u_{1}-\nabla u_{2}\right)^{2} d x d y
$$

for all $(\eta, \tau) \in U_{a d}=\left(H_{00}^{\frac{1}{2}}(\mathcal{O})\right)^{\prime} \times H_{00}^{\frac{1}{2}}(\mathcal{O})$. When it exists, the optimal control is given by:

$$
(\varphi, t)=\arg \min \left\{E(\eta, \tau) ;(\eta, \tau) \in U_{a d}\right\}
$$

The spaces $\left(H_{00}^{\frac{1}{2}}(\mathcal{O})\right)^{\prime}$ and $H_{00}^{\frac{1}{2}}(\mathcal{O})$ are equipped, respectively, with the following inner product (see [19]):

$$
(u, v)_{\left(H_{00}^{\frac{1}{2}}(\mathcal{O})\right)^{\prime}}=\int_{\Gamma_{a}}\left(-\Delta_{\Gamma_{a}}\right)^{-\frac{1}{4}} u\left(-\Delta_{\Gamma_{a}}\right)^{-\frac{1}{4}} v d y=\int_{\Gamma_{a}} u\left(-\Delta_{\Gamma_{a}}\right)^{-\frac{1}{2}} v d y,
$$

for all $u, v \in\left(H_{00}^{\frac{1}{2}}(\mathcal{O})\right)^{\prime}$, and

$$
(f, g)_{H_{00}^{\frac{1}{2}}(\mathcal{O})}=\int_{\Gamma_{a}}\left(-\Delta_{\Gamma_{a}}\right)^{\frac{1}{4}} f\left(-\Delta_{\Gamma_{a}}\right)^{\frac{1}{4}} g d y=\int_{\Gamma_{a}} f\left(-\Delta_{\Gamma_{a}}\right)^{\frac{1}{2}} g d y
$$

for all $f, g \in H_{00}^{\frac{1}{2}}(\mathcal{O})$. 


\section{Factorization of boundary value problems}

The method was presented in [13]. We consider the problem of Poisson in the open cylindrical domain $\Omega$ defined by:

$$
\left(\mathcal{P}_{1}\right)\left\{\begin{array}{lll}
-\Delta u=f & \text { in } & \Omega, \\
u=0 & \text { on } & \Sigma, \\
u=g & \text { on } & \Gamma_{a}, \\
-\frac{\partial u}{\partial x}=u_{0} & \text { on } & \Gamma_{0} .
\end{array}\right.
$$

We apply the method of invariant embedding developped by R. Bellman in [3]. In control theory this method gives the optimal feedback : one embeds the problem of control in a family of similar problems defined between the present and the final time. In our work this invariant embeding is spatial: we embed the problem $\left(\mathcal{P}_{1}\right)$ in a family of similar problems $\left(\mathcal{P}_{s, k}\right)$ defined on $\left.\Omega_{s}=\right] 0, s[\times \mathcal{O}$. For every problem we impose a boundary condition of Dirichlet $\left.u\right|_{\Gamma_{s}} ^{s}=k \Gamma_{s}=\{s\} \times \mathcal{O}$.

$$
\left(\mathcal{P}_{s, k}\right)\left\{\begin{array}{llc}
-\Delta u^{s}=f & \text { in } & \Omega_{s} \\
u^{s}=0 & \text { on } & \Sigma_{s} \\
u^{s}=k & \text { on } & \Gamma_{s} \\
-\frac{\partial u^{s}}{\partial x}=u_{0} & \text { on } & \Gamma_{0} .
\end{array}\right.
$$

Let $u^{s}=u^{s}(k)$ be the solution of $\left(\mathcal{P}_{s, k}\right)$. It's clear that $\left(\mathcal{P}_{1}\right)$ is exactly $\left(P_{s, k}\right)$ with $s=a$ and $k=g$. The calculation is now similar to the one developped by Lions in [20] to find the optimal feedback for a problem of optimal control for a parabolic equation. For every $s \in] 0, a]$ we define the Dirichlet-Neumann mapping (D-N), $P(s)$ by $P(s) k=\left.\frac{\partial \gamma_{s}}{\partial x}\right|_{\mid \Gamma_{s}}$, where $\gamma_{s}$ is the solution of

$$
\left\{\begin{array}{lll}
-\Delta \gamma_{s}=0 & \text { in } & \Omega_{s} \\
\gamma_{s}=0 & \text { on } & \Sigma_{s} \\
\gamma_{s}=k & \text { on } & \Gamma_{s} \\
-\frac{\partial \gamma_{s}}{\partial x}=0 & \text { on } & \Gamma_{0}
\end{array}\right.
$$

Let $w$ be defined by $w(s)=\left.\frac{\partial \beta_{s}}{\partial x}\right|_{\Gamma_{s}}$, where $\beta_{s}$ is the solution of

$$
\left\{\begin{array}{lll}
-\Delta \beta_{s}=f & \text { in } & \Omega_{s} \\
\beta_{s}=0 & \text { on } & \Sigma_{s} \\
\beta_{s}=0 & \text { on } & \Gamma_{s} \\
-\frac{\partial \beta_{s}}{\partial x}=u_{0} & \text { on } & \Gamma_{0}
\end{array}\right.
$$

and $w(0)=-u_{0}$.

The linearity of $\left(\mathcal{P}_{s, k}\right)$ yields $u^{s}=\beta_{s}+\gamma_{s}$. So by the well-posedness of the problem on 
$\gamma$ and the continuity of the trace mapping

$$
\left.\frac{\partial u^{s}}{\partial x}\right|_{\Gamma_{s}}=P(s) k+w
$$

It is shown in $([13])$ that for every $s \in] 0, a], P(s): H_{00}^{\frac{1}{2}}(\mathcal{O}) \longrightarrow H_{00}^{\frac{1}{2}}(\mathcal{O})^{\prime}$ is a coercive, self-adjoint, linear continuous operator and $w(s)=\frac{\partial \beta_{s}}{\partial x} \mid \Gamma_{s} \in H_{00}^{\frac{1}{2}}(\mathcal{O})^{\prime}$.

We consider for $x \leq s$ the restriction of $u^{s}(k)$ at $\Omega_{x}$. It is solution of the problem $\left(\mathcal{P}_{x, u^{s}(x, k)}\right)$.

By the same calculation as previously we have the relation:

$$
\frac{\partial u^{s}}{\partial x}(x, k)=P(x) u^{s}(x, k)+w(x)
$$

On a formal way, we take the derivative of (5) with respect to $x$ (stressing the particular role plaid by $x$ we denote it by $\frac{d}{d x}$ ) and using the equation satisfied by $u^{s}$,

$$
\frac{d^{2} u^{s}}{d x^{2}}=-\Delta_{y} u^{s}-f=\frac{d P}{d x} u^{s}+P \frac{d u^{s}}{d x}+\frac{d w}{d x}
$$

Subtituting $\frac{d u^{s}}{d x}$ from (5) and for $x=s$ we find

$$
\left(\Delta_{y}+\frac{d P}{d x}(s)+P^{2}(s)\right) k+P(s) w(s)+\frac{d w(s)}{d x}+f(s)=0 .
$$

In this calculation $k$ and $s$ are arbitrary and we deduce:

$$
\left.\left.\frac{d P}{d x}+P^{2}=-\Delta_{y}, \text { for } x \in\right] 0, a\right]
$$

For the residual term

$$
\left.\left.\frac{d w}{d x}+P w=-f, \text { for } x \in\right] 0, a\right]
$$

One can extend by continuity this argument to $x=0$. Writing the relation (5) at $x=0$, and by using the fact that $u(0)$ is arbitrary, we get the initial conditions for $P$ and $w$

$$
P(0)=0, \quad w(0)=-u_{0}
$$

Adding the condition on $\Gamma_{a}$ for $u$, we summarize the result: an equivalent formulation of $\left(\mathcal{P}_{1}\right)$ is

$$
\begin{cases}\frac{d P}{d x}+P^{2}=-\Delta_{y}, & P(0)=0 \\ \frac{d w}{d x}+P w=0, & w(0)=-u_{0} \\ -\frac{d u}{d x}+P u=-w, & u(a)=g\end{cases}
$$

The system (9) is uncoupled because one can integrate the two first equations in $x$ from 0 to $a$ to find $P$ and $w$, then $u$ is obtained by backward integration. In a formal way, having $P$, one can factorize $\left(\mathcal{P}_{1}\right)$ in

$$
-\left(\frac{d}{d x}+P\right)\left(\frac{d}{d x}-P\right) u=f .
$$




\section{The factorization method as a tool to the boundary data recovering}

In this section we show that applying the factorization method to the states $u_{1}, u_{2}$, we can express the cost function explicitly in terms of the controls $\tau, \eta$. We embed the control problem in a family of similar problems defined on $\Omega_{s}$. For (1) we impose a Neumann boundary condition on $\Gamma_{s}:\left.\frac{d u_{1}^{s}}{d x}\right|_{\Gamma_{s}}=\alpha$, and for (2) a Dirichlet boundary condition $:\left.u_{2}\right|_{\Gamma_{s}} ^{s}=\beta$. The originality of the approach lies in the choice of the embedding: the controls $\tau, \eta$ do not show up in the problems set in $\Omega_{s}$

$$
\begin{aligned}
& \begin{cases}\Delta u_{1}^{s}=0 & \text { in } \Omega_{s}, \\
u_{1}^{s}=0 & \text { on } \Sigma_{s}, \\
u_{1}^{s}=T & \text { on } \Gamma_{0}, \quad \frac{\partial u_{1}^{s}}{\partial n}=\alpha \quad \text { on } \Gamma_{s},\end{cases} \\
& \begin{cases}\Delta u_{2}^{s}=0 & \text { in } \Omega_{s}, \\
u_{2}^{s}=0 & \text { on } \Sigma_{s}, \\
\frac{\partial u_{2}^{s}}{\partial n}=\Phi & \text { on } \Gamma_{0}, \quad u_{2}^{s}=\beta \quad \text { on } \Gamma_{s} .\end{cases}
\end{aligned}
$$

We consider the Neumann-Dirichlet(N-D) mapping for $u_{1}:\left.\alpha \longmapsto u_{1}^{s}\right|_{\Gamma_{s}}$ and the Dirichlet-Neumann (D-N) for $u_{2}:\left.\beta \longmapsto \frac{d u_{2}^{s}}{d x}\right|_{\Gamma_{s}}$. From section 3 we have

$$
\left.\frac{d u_{2}^{s}}{d x}\right|_{\Gamma_{s}}=P(s) \beta+w_{2}(s)
$$

with $P(s) \in \mathcal{L}\left(H_{00}^{\frac{1}{2}}(\mathcal{O}),\left(H_{00}^{\frac{1}{2}}(\mathcal{O})\right)^{\prime}\right), P(0)=0$ and $w_{2}(0)=-\Phi$. The invariant embeding of section 3 leads to

$$
\begin{aligned}
& \frac{d P}{d x}+P^{2}=-\Delta_{y}, \quad P(0)=0 \\
& \frac{d w_{2}}{d x}+P w_{2}=0, \quad w_{2}(0)=-\Phi .
\end{aligned}
$$

Then $u_{2}$ is found by backward integration of

$$
\frac{d u_{2}}{d x}=P u_{2}+w_{2}, \quad u_{2}(a)=\tau
$$

We can treat the (N-D) operator $Q$ in a similar way, for $u_{1}$ satisfying (11),

$$
u_{1}^{s}(s)=Q(s) \alpha+w_{1}(s), \quad Q(s) \in \mathcal{L}\left(\left(H_{0,0}^{\frac{1}{2}}(\mathcal{O})\right)^{\prime}, H_{0,0}^{\frac{1}{2}}(\mathcal{O})\right) .
$$

Considering the invariant embeding for $x \leq s$ we get

$$
u_{1}^{s}(x)=Q(s) \frac{d u_{1}^{s}}{d x}(x)+w_{1}(x) .
$$

Taking the derivative with respect to $x$

$$
\frac{d u_{1}^{s}}{d x}=\frac{d Q}{d x} \frac{d u_{1}^{s}}{d x}+Q \frac{d^{2} u_{1}^{s}}{d x^{2}}+\frac{d w_{1}}{d x} .
$$


Substituting $u_{1}^{s}$, using (11), taking $x=s$ and using the fact that $\alpha$ is arbitrary we get

$$
\begin{aligned}
& \frac{d Q}{d x}-Q \Delta_{y} Q=I, \quad Q(0)=0, \\
& \frac{d w_{1}}{d x}-Q \Delta_{y} w_{1}(x)=0, \quad w_{1}(0)=T .
\end{aligned}
$$

After solving these equations we find $u_{1}$ by backward integration of the implicit differential equation

$$
u_{1}=Q \frac{d u_{1}}{d x}+w_{1}, \quad u_{1}(a)=Q(a) \eta+w_{1}(a) .
$$

\subsection{Solving the optimal control problem}

Let us show now that the energy functional $E$ can be expressed directly in terms of the control variable $\eta$ and $\tau$ using the operators $P$ and $Q$. So there is no need of introducing an adjoint state to derive the optimality condition. Saying it in another way, the classical decoupling of the optimality system between state and adjoint state using a Riccati equation is done here at the same time as the factorization of the state equation.

From now on we will denote $P=P(a)$ and $Q=Q(a)$. Let $A$ be the matrix operator

$$
A=\left(\begin{array}{cc}
Q & -Q P \\
-P Q & P
\end{array}\right) \text {. }
$$

Let us denote $[\eta, \tau]$ the row vector with component $\eta$ and $\tau$. Let us remark that in relation to the ill-posedness of the Cauchy problem, the operator $A$ is not coercive.

Proposition 1 The cost functional E can be written equivalently

$$
E(\eta, \tau)=C+[\eta, \tau] A[\eta, \tau]^{\prime}-2 \int_{\Gamma_{a}}\left(w_{1} P \tau+w_{2} Q \eta\right) d y
$$

where $C$ is a constant not depending on $\eta$ and $\tau$. If a minimum $(\varphi, t)$ of $E$ is reached it satisfies

$$
A[\varphi, t]^{\prime}=\left[Q w_{2}, P w_{1}\right]^{\prime} .
$$

Proof

Let $\alpha$ and $\beta$ be the solutions of

$$
\left\{\begin{array}{lll}
\Delta \alpha=0 & \text { in } & \Omega \\
\alpha=0 & \text { on } & \Sigma \\
\alpha=T & \text { on } & \Gamma_{0} \\
\frac{\partial \alpha}{\partial n}=0 & \text { on } & \Gamma_{a}
\end{array}\right.
$$




$$
\left\{\begin{array}{llc}
\Delta \beta=0 & \text { in } & \Omega, \\
\beta=0 & \text { on } & \Sigma, \\
\frac{\partial \beta}{\partial n}=\Phi & \text { on } & \Gamma_{0} \\
\beta=0 & \text { on } & \Gamma_{a} .
\end{array}\right.
$$

Using Green's formula and (1), (2)

$$
\begin{aligned}
E(\eta, \tau) & =\int_{\Omega}\left(\nabla u_{1}-\nabla u_{2}\right)^{2} d x d y \\
& =\int_{\Gamma_{0}}\left(\frac{\partial u_{1}}{\partial n}-\frac{\partial u_{2}}{\partial n}\right)\left(u_{1}-u_{2}\right) d y+\int_{\Gamma_{a}}\left(\frac{\partial u_{1}}{\partial n}-\frac{\partial u_{2}}{\partial n}\right)\left(u_{1}-u_{2}\right) d y \\
& =\int_{\Gamma_{0}}\left(\frac{\partial u_{1}}{\partial n}-\Phi\right)\left(T-u_{2}\right) d y+\int_{\Gamma_{a}}\left(\frac{\partial u_{1}}{\partial n}-\frac{\partial u_{2}}{\partial n}\right)\left(u_{1}-u_{2}\right) d y \\
& =\int_{\Gamma_{0}}\left(-\Phi T+\frac{\partial u_{1}}{\partial n} T+\Phi u_{2}-\frac{\partial u_{1}}{\partial n} u_{2}\right) d y+\int_{\Gamma_{a}}\left(\eta-\frac{\partial u_{2}}{\partial n}\right)\left(u_{1}-\tau\right) d y
\end{aligned}
$$

Using the full Green's formula for the term in $\frac{\partial u_{1}}{\partial n} u_{2}$

$$
\begin{aligned}
E(\eta, \tau)= & \int_{\Gamma_{0}}\left(-2 \Phi T+\frac{\partial u_{1}}{\partial n} \alpha+\frac{\partial \beta}{\partial n} u_{2}\right) d y+\int_{\Gamma_{a}}\left(\eta \tau-u_{1} \frac{\partial u_{2}}{\partial n}\right) d y+ \\
& +\int_{\Gamma_{a}}\left(\eta-\frac{\partial u_{2}}{\partial n}\right)\left(u_{1}-\tau\right) d y
\end{aligned}
$$

and for the terms in $\frac{\partial u_{1}}{\partial n} \alpha$ and $\frac{\partial \beta}{\partial n} u_{2}$

$$
\begin{aligned}
E(\eta, \tau)= & -2 \int_{\Gamma_{0}} \Phi T d y+\int_{\Gamma_{0}}\left(T \frac{\partial \alpha}{\partial n}+\beta \Phi\right) d y-\int_{\Gamma_{a}}\left(\eta \alpha+\frac{\partial \beta}{\partial n} \tau\right) d y \\
& +\int_{\Gamma_{a}}\left(\eta \tau-u_{1} \frac{\partial u_{2}}{\partial n}\right) d y+\int_{\Gamma_{a}}\left(\eta-\frac{\partial u_{2}}{\partial n}\right)\left(u_{1}-\tau\right) d y \\
= & -2 \int_{\Gamma_{0}} \Phi T d y+\int_{\Gamma_{0}}\left(T \frac{\partial \alpha}{\partial n}+\beta \Phi\right) d y+\int_{\Gamma_{a}}\left(\eta\left(u_{1}-\alpha\right)+\left(\frac{\partial u_{2}}{\partial n}-\frac{\partial \beta}{\partial n}\right) \tau\right) d y \\
& -2 \int_{\Gamma_{a}} u_{1} \frac{\partial u_{2}}{\partial n} d y
\end{aligned}
$$

From (15), (18) we have

$$
\begin{aligned}
& u_{1}(a)=Q(a) \frac{\partial u_{1}}{\partial n}(a)+w_{1}(a)=Q(a) \eta+w_{1}(a) \\
& \frac{\partial u_{2}}{\partial n}(a)=P(a) u_{2}(a)+w_{2}(a)=P(a) \tau+w_{2}(a) .
\end{aligned}
$$

Then from the definition of $\alpha$ and $\beta$ we have

$$
\alpha(a)=w_{1}(a), \quad \frac{\partial \beta}{\partial n}(a)=w_{2}(a) .
$$


Then

$$
\begin{aligned}
E(\eta, \tau)= & -2 \int_{\Gamma_{0}} \Phi T d y+\int_{\Gamma_{0}}\left(T \frac{\partial \alpha}{\partial n}+\beta \Phi\right) d y+\int_{\Gamma_{a}}(\eta Q \eta+\tau P \tau) d y \\
& -2 \int_{\Gamma_{a}}\left(Q \eta+w_{1}\right)\left(P \tau+w_{2}\right) d y
\end{aligned}
$$

The relation at the minimum results from the quadratic form of $E$, its convexity and the selfadjointness of $P$ and $Q$.

Proposition 2 Let $w_{1}$ and $w_{2}$ be given by (17) and (14). The relation (19) at the minimum of $E$ is equivalent to

$$
(I-Q P) t=Q w_{2}+w_{1} \text { and }(I-P Q) \varphi=P w_{1}+w_{2} .
$$

The compatibility of the data $(\Phi, T)$ with respect to the Cauchy problem, is given by one of the three following assertions that are equivalent

(i) $\left(Q w_{2}, P w_{1}\right)^{\prime} \in \Im(A)$,

(ii) $Q w_{2}+w_{1} \in \Im(I-Q P)$,

(iii) $w_{2}+P w_{1} \in \Im(I-P Q)$.

Proof

The relation (19) reads

$$
\left\{\begin{array}{l}
Q \varphi-Q P t=Q w_{2} \\
P t-P Q \varphi=P w_{1}
\end{array}\right.
$$

By the coerciveness of $P$ and the Lax-Milgram Theorem, $P$ is an isomorphism from $H_{00}^{\frac{1}{2}}\left(\Gamma_{a}\right)$ to $\left(H_{00}^{\frac{1}{2}}\left(\Gamma_{a}\right)\right)^{\prime}$. Then the second equation $(24)$ yields

$$
t=Q \varphi+w_{1} .
$$

In the same way the first equation (24) yields

$$
\varphi=P t+w_{2} \text {. }
$$

Then (23) is obtained by the combination $Q(26)+(25)$ and $(26)+P(25)$.

Reciprocally, assume (23) is satisfied. Let us show that $I-Q P$ is injective. Suppose there exists $\tau$ such that $(I-Q P) \tau=0$. This means that considering the homogeneous problems $(\Phi=T=0)(1)$, (2) we get

$$
\left.u_{1}(\eta)\right|_{\Gamma_{a}}=\left.u_{2}\right|_{\Gamma_{a}}, \quad \text { for } \eta=\left.\frac{\partial u_{2}}{\partial n}\right|_{\Gamma_{a}} .
$$

Then by Holmgren's Theorem, $u_{1}=u_{2}$ and by the boundary conditions at $\Gamma_{0}$ and Holmgren's Theorem once again

$$
u_{1}=u_{2}=0
$$

and so $\tau=0$. Similarly $I-P Q$ is injective

From (23) the combination $-P(23)_{1}+(23)_{2}$ yields

$$
(I-P Q)(\varphi-P t)=(I-P Q) w_{2}
$$


And so we recover (26) and in the same way we can recover (25). Then (19) is obtained by a linear combination of (26) and(25).

Let us now turn to the compatibility condition for the data $(\Phi, T)$. If $\left(Q w_{2}, P w_{1}\right)^{\prime} \in$ $\Im(A)$ then there exists $(\varphi, t) \in\left(H_{00}^{\frac{1}{2}}\left(\Gamma_{a}\right)\right)^{\prime} \times H_{00}^{\frac{1}{2}}\left(\Gamma_{a}\right)$ solution of the system (24). Let us define $u_{1}$ and $u_{2}$ as previously with $\left.u_{2}\right|_{\Gamma_{a}}=t$ and $\left.\frac{\partial u_{1}}{\partial n}\right|_{\Gamma_{a}}=\varphi$. Then the second relation (24) gives

$$
\begin{aligned}
\frac{\partial u_{2}}{\partial n}-w_{2} & =P Q \frac{\partial u_{1}}{\partial n}+P w_{1} \\
& =P u_{1}
\end{aligned}
$$

Then $P(a) u_{1}(a)=P(a) u_{2}(a)$, and by the coerciveness of $P$ and Lax Milgram Theorem:

$$
u_{1}(a)=u_{2}(a)
$$

Similarly from the first relation (24)

$$
\left.\frac{\partial u_{1}}{\partial n}\right|_{\Gamma_{a}}=\left.\frac{\partial u_{2}}{\partial n}\right|_{\Gamma_{a}}
$$

This implies that $(\varphi, t)$ is the solution of the inverse problem of data completion and then $(\Phi, T)$ are compatible.

Reciprocally if $(\Phi, T)$ are compatible then the inverse problem has a unique solution $(\varphi, t)$ that is the minimum of the function $E$. Then Proposition 1 gives

$$
A[\varphi, t]^{\prime}=\left[Q w_{2}, P w_{1}\right]^{\prime}
$$

Let us show that assertion 2) implies 3). Let $Q w_{2}+w_{1} \in \Im(I-Q P)$ and $t$ such that $t-Q P t=Q w_{2}+w_{1}$. Then $t-w_{1}=Q P t+Q w_{2}$. Let us set $\varphi=P t+w_{2}$. We have

$$
Q \varphi=Q P t+Q w_{2}=t-w_{1}
$$

Multiplying by $P$

$$
P Q \varphi=P t-P w_{1}=\varphi-w_{2}-P w_{1}
$$

that is

$$
w_{2}+P w_{1} \in \Im(I-P Q) .
$$

By a similar calculation 3) implies 2).

From the previous calculation if (24) is satisfied (23) is also and so 1) implies 2) and 3). Also we already showed that reciprocally 2) and 3) imply 1).

\section{Link with the Steklov-Poincaré operator approach}

In this part we present a link between the previous approach and the calculation of [2] using the Steklov-Poincaré operator. For all $(\Phi, T) \in\left(H_{00}^{\frac{1}{2}}\left(\Gamma_{a}\right)\right)^{\prime} \times H_{00}^{\frac{1}{2}}\left(\Gamma_{a}\right)$ and for all $\tau \in H_{00}^{\frac{1}{2}}\left(\Gamma_{a}\right)$, we consider the problem $(1)$, (2), where we control $u_{1}$ with a Dirichlet 
condition $u_{1}=\tau$ on $\Gamma_{a}$ in order to be in the same framework than [2], that is $u_{1}$ now satisfies

$$
\begin{cases}\Delta u_{1}=0 & \text { in } \Omega, \\ u_{1}=0 & \text { on } \Sigma, \\ u_{1}=T & \text { on } \Gamma_{0}, \quad u_{1}=\tau \quad \text { on } \Gamma_{a}\end{cases}
$$

We denote the solutions of $(27),(2)$ by $u_{1}(\tau, T)$ and $u_{2}(\tau, \Phi)$ to recall the dependencies with respect to the control and the data.

We denote $u_{1}(\tau, 0)\left(\operatorname{resp} u_{2}(\tau, 0)\right)$ by $u_{1}(\tau)\left(\operatorname{resp} u_{2}(\tau)\right)$ and $u_{1}(0, T)$ (resp $u_{2}(0, \Phi)$ by $u_{1}^{0}(\tau)$ (resp $\left.u_{2}^{0}(\tau)\right)$. We introduce now the bilinear forms $s(.,),. s_{D}(.,$.$) and s_{N}(.,$.$) in H_{00}^{\frac{1}{2}}\left(\Gamma_{a}\right) \times H_{00}^{\frac{1}{2}}\left(\Gamma_{a}\right)$ and the linear form $l$ in $H_{00}^{\frac{1}{2}}\left(\Gamma_{a}\right)$ defined for all $\tau, \delta \in H_{00}^{\frac{1}{2}}\left(\Gamma_{a}\right)$ by :

$$
\begin{gathered}
s(\tau, \delta)=s_{D}(\tau, \delta)-s_{N}(\tau, \delta)=\int_{\Omega} \nabla u_{1}(\tau) \nabla u_{1}(\delta) d x d y-\int_{\Omega} \nabla u_{2}(\tau) \nabla u_{2}(\delta) d x d y, \\
l(\delta)=-\int_{\Omega} \nabla u_{1}^{0}(T) \nabla u_{1}(\delta) d x d y+\int_{\Omega} \nabla u_{2}^{0}(\Phi) \nabla u_{2}(\delta) d x d y-\left(\Phi, u_{2}(\delta)\right)_{L^{2}\left(\Gamma_{0}\right)} .
\end{gathered}
$$

The boundary data recovery amounts to finding $t \in H_{00}^{\frac{1}{2}}\left(\Gamma_{a}\right)$ such that $\nabla u_{1}(t)=\nabla u_{2}(t)$ on the face $\Gamma_{a}$. This is equivalent to finding $t \in H_{00}^{\frac{1}{2}}\left(\Gamma_{a}\right)$ verifying

$$
s(t, \delta)=l(\delta) \quad \text { for all } \quad \delta \in H_{00}^{\frac{1}{2}}\left(\Gamma_{a}\right) .
$$

Proposition 3 The bilinear forms $s, s_{D}$ and $s_{N}$ and the linear form $l$ are related to the operators solution of the Riccati equations (13), (16) and the remainder functions $w_{1}$ and $w_{2}$ by

$$
\begin{gathered}
s_{N}(\tau, \delta)=(P \tau, \delta), \quad s_{D}(\tau, \delta)=\left(Q^{-1} \tau, \delta\right), \\
s(\tau, \delta)=\left(\left(Q^{-1}-P\right) \tau, \delta\right), \quad l(\delta)=\left(Q^{-1} w_{1}+w_{2}, \delta\right),
\end{gathered}
$$

for all $\tau, \delta \in H_{00}^{\frac{1}{2}}\left(\Gamma_{a}\right)$.

Proof

By Green's formula

$$
\begin{aligned}
s_{D}(\tau, \delta) & =\int_{\Omega} \nabla u_{1}(\tau) \nabla u_{1}(\delta) d x d y \\
& =\int_{\Gamma_{a}} \frac{\partial u_{1}}{\partial n}(\tau) \delta d y \\
& =\int_{\Gamma_{a}} Q^{-1} \tau \delta d y \\
s_{N}(\tau, \delta) & =\int_{\Omega} \nabla u_{2}(\tau) \nabla u_{2}(\delta) d x d y \\
& =\int_{\Gamma_{a}} \frac{\partial u_{2}}{\partial n}(\tau) \delta d y \\
& =\int_{\Gamma_{a}} P \tau \delta d y
\end{aligned}
$$




$$
\begin{aligned}
s(\tau, \delta) & =s_{D}(\tau, \delta)-s_{N}(\tau, \delta) \\
& =\int_{\Gamma_{a}}\left(Q^{-1}-P\right) \tau \delta d y \\
l(\delta)= & -\int_{\Omega} \nabla u_{1}^{0}(T) \nabla u_{1}(\delta) d x d y+\int_{\Omega} \nabla u_{2}^{0}(\Phi) \nabla u_{2}(\delta) d x d y-\left(\Phi, u_{2}(\delta)\right)_{L^{2}\left(\Gamma_{0}\right)} \\
= & -\int_{\Gamma_{0}} T \frac{\partial u_{1}}{\partial n}(\delta) d y-\int_{\Gamma_{0}} \Phi u_{2}(\delta) d y .
\end{aligned}
$$

From (20), (21), using the full Green's formula and the relation (22) one gets

$$
\begin{aligned}
l(\delta) & =-\int_{\Gamma_{0}} \alpha \frac{\partial u_{1}}{\partial n}(\delta) d y-\int_{\Gamma_{0}} \frac{\partial \beta}{\partial n} u_{2}(\delta) d y \\
& =\int_{\Gamma_{a}} w_{1} \nabla u_{1}(\delta) d y+\int_{\Gamma_{a}} w_{2} \delta d y .
\end{aligned}
$$

We shall denote by $S(x)$ the operator $S(x)=Q^{-1}(x)-P(x)$ and so

$$
s(\tau, \delta)=(S \tau, \delta)
$$

Remark 1 The definition of $u_{1}$ given by (27) is the same than the one of (1) if $\tau$ and $\eta$ are linked by

$$
\tau=Q(a) \eta+w_{1} .
$$

In this case the second relation (24) is automatically satisfied which proves that the two formulations are equivalent.

Remark 2 These relations may be particularly usefull if one wants to study the sensitivity of the data completion problem to the size a of the domain because the derivatives of $P, Q, w_{1}$ and $w_{2}$ with respect to a are given by (13), (16), (17), (14).

\section{Generalization}

In this section we extend the calculations of section 4 with two controls $\eta, \tau$ to the case where the two states $u_{1}, u_{2}$ are controlled through Robin conditions on $\Gamma_{a}$. We consider the following two mixed well posed problems for both $\eta$ and $\tau$ in $H_{00}^{\frac{1}{2}}\left(\Gamma_{a}\right)^{\prime}, r_{1}>0$ and $r_{2}>0$.

$$
\begin{aligned}
& \begin{cases}\Delta u_{1}=0 & \text { in } \Omega, \\
u_{1}=0 & \text { on } \Sigma, \\
u_{1}=T & \text { on } \Gamma_{0}, \quad \frac{\partial u_{1}}{\partial n}+r_{1} u_{1}=\eta+r_{1} \tau \quad \text { on } \Gamma_{a}\end{cases}
\end{aligned}
$$

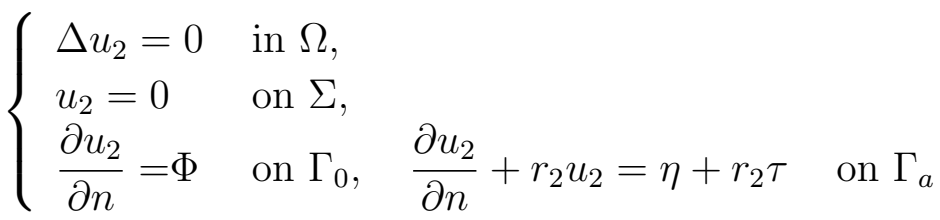


Here again, the next step is to build an error functional on the two fields by

$$
E_{r_{1} r_{2}}(\eta, \tau)=\int_{\Omega}\left(\nabla u_{1}-\nabla u_{2}\right)^{2} d x d y
$$

with, $u_{1}$ and $u_{2}$ being the solution of (28) and (29). If the minimum exists we define

$$
(\varphi, t)=\arg \min E_{r_{1} r_{2}}(\eta, \tau)
$$

Indeed, $u_{1}$ and $u_{2}$ are obviously equal when the pair $(\Phi, T)$ is compatible and one gets on $\Gamma_{a}$

$$
u_{1}=u_{2}=t, \quad \frac{\partial u_{1}}{\partial n}=\frac{\partial u_{2}}{\partial n}=\varphi
$$

Let us set

$$
\begin{array}{cc}
Q_{1}=\left(I+r_{1} Q\right)^{-1}, & P_{1}=\left(P+r_{2} I\right)^{-1}, \\
X_{1}=Q Q_{1}^{2}+P P_{1}^{2}-2 Q P Q_{1} P_{1}, & X_{2}=r_{1}^{2} Q Q_{1}^{2}+r_{2}^{2} P P_{1}^{2}-2 r_{1} r_{2} Q P Q_{1} P_{1}, \\
X_{3}=r_{1} Q Q_{1}^{2}+r_{2} P P_{1}^{2}-\left(r_{1}+r_{2}\right) Q P Q_{1} P_{1}, & X_{4}=r_{1} Q_{1}\left(Q_{1}-I-r_{1} Q Q_{1}\right)-2 r_{2} P P_{1} Q_{1}, \\
X_{5}=r_{2} P_{1}\left(r_{2} P_{1}-I-P P_{1}\right)-2 r_{1} r_{2} Q Q_{1} P_{1} & X_{6}=Q_{1}\left(Q_{1}-I\right)-R_{1} Q_{1}^{2} Q-2 P P_{1} Q_{1} \\
X_{7}=P_{1}\left(r_{2} P_{1}-I-P P_{1}\right)-2 r_{2} P_{1} Q Q_{1} . &
\end{array}
$$

Then setting

$$
A_{r_{1} r_{2}}=\left(\begin{array}{cc}
X_{1} & X_{3} \\
X_{3} & X_{2}
\end{array}\right),
$$

by the same calculations as in section 4 we get

$$
E_{r_{1} r_{2}}(\eta, \tau)=C+[\eta, \tau] A_{r_{1} r_{2}}[\eta, \tau]^{\prime}+\int_{\Gamma_{a}}\left(\left(X_{4} w_{1}+X_{5} w_{2}\right) \tau+\left(X_{6} w_{1}+X_{7} w_{2}\right) \eta\right) d y
$$

With this formulation we can recover various situations: taking $r_{1}=0, r_{2}=+\infty$ we recover the formulation (1), (2) and

$$
E_{r_{1} r_{2}}(\eta, \tau)=E(\eta, \tau)
$$

Taking $r_{1}=r_{2}=+\infty$ we have

$$
\left.u_{1}\right|_{\Gamma_{a}}=\left.u_{2}\right|_{\Gamma_{a}}=\tau
$$

so this is the framework of [2] recalled in section 5 . Then

$$
E_{r_{1} r_{2}}(\eta, \tau)=\left(\left(Q^{-1}-P\right) \tau, \tau\right)-2\left(Q^{-1} w_{1}+w_{2}, \tau\right)+C
$$

The case $r_{1}=r_{2}<+\infty$ corresponds to a Steklov-Poincaré approach as in [2] but with a Robin condition control. In the domain decomposition method it is known to be numerically more efficient. 


\section{Explicit Formulae}

\subsection{Determination of the interfacial operators}

In this section we take full advantage of the cylindrical geometry of $\Omega$ to derive explicit formulae for the Dirichlet-Neumann $P(a)$ and Neumann-Dirichlet $Q(a)$ operators at the face $\Gamma_{a}$.

Proposition 4 Let $U(x)$ be the $C^{0}$ semi-group with generator $-2\left(-\Delta_{y}\right)^{\frac{1}{2}}$. For cylindrical domains $\Omega$ the solution of (13) is explicitly given by

$$
P(x)=\left(-\Delta_{y}\right)^{\frac{1}{2}}(I-U(x))(I+U(x))^{-1} .
$$

The solution $Q(x)$ of (16) is then given by

$$
Q(x)=\left(-\Delta_{y}\right)^{-1} P(x) .
$$

The operators $P$ and $Q$ commute and $R$ is given by

$$
R(x)=I-P(x) Q(x)=I-Q(x) P(x)=4 U(x)(U(x)+I)^{-2} .
$$

Proof

The formula (30) is proved in [5] by using a Yosida regularization. From this formula, $P$ being a function of $-\Delta_{y}$ commutes with it and any of its power. Let us multiply (13) by $\left(-\Delta_{y}\right)^{-1}$. We deduce that $\left(-\Delta_{y}\right)^{-1} P$ is a solution of $(16)$, which has a unique solution so that

$$
Q=\left(-\Delta_{y}\right)^{-1} P=\left(-\Delta_{y}\right)^{-\frac{1}{2}}(I-U(x))(I+U(x))^{-1} .
$$

Then $R=I-P Q$ is given by

$$
\begin{aligned}
R(x) & =I-\left(-\Delta_{y}\right)^{-1} Q^{2}(x) \\
& =4 U(x)(U(x)+I)^{-2} .
\end{aligned}
$$

Similarly $S(x)=Q^{-1}(x)-P(x)$ is given by

$$
S(x)=4\left(-\Delta_{y}\right)^{\frac{1}{2}} U(x)\left(I-U^{2}(x)\right)^{-1} .
$$

\subsection{Behaviour with respect to the length of the cylinder}

We use here the explicit formulae obtained in section (7) to study the dependence of the ill-posedness of the problem with respect to the length of the cylinder. If the data $(\Phi, T)$ are compatible the solution $(\varphi, t)$ of the problem $\left(\mathcal{P}_{0}\right)$ is given by

$$
\left(S(a)^{-1} w_{2}(a)+R(a)^{-1} w_{1}(a), R(a)^{-1} w_{2}(a)-\Delta_{y} S(a)^{-1} w_{1}(a)\right) .
$$

Let $\left\{\alpha_{n}, n \in \mathbb{N}\right\}, \alpha_{n}>0$ be the spectrum of $\left(-\Delta_{y}\right)^{\frac{1}{2}}$. The sequence $\left(\alpha_{n}\right)$ increases to infinity. Then the spectrum of $R(a)$ is given by

$$
\left\{\frac{4 e^{-2 \alpha_{n} a}}{\left(1+e^{-2 \alpha_{n} a}\right)^{2}}, \quad n \in \mathbb{N}\right\}
$$


that of $S(a)$ is given by

$$
\left\{\alpha_{n} \frac{4 e^{-2 \alpha_{n} a}}{1-e^{-4 \alpha_{n} a}}, \quad n \in \mathbb{N}\right\}
$$

and that of $\left(-\Delta_{y}\right)^{-1} S(a)$ by

$$
\left\{\frac{4 e^{-2 \alpha_{n} a}}{\alpha_{n}\left(1-e^{-4 \alpha_{n} a}\right)}, \quad n \in \mathbb{N}\right\} .
$$

One notices that these sequences decrease exponentially to 0 , when $\mathrm{n}$ goes to infinity, and that illustrates the ill-posedness of the inverse problem $\left(\mathcal{P}_{0}\right)$.

We also notice that if the length of the cylinder increases, the speed of convergence of the sequences to zero increases. So these formulae allow to quantify the ill-posedness of the inverse problem according to the length of the cylinder (i.e. the distance between the surface where the data are overdetermined and the one where they are missing).

\subsection{Regularizing the data completion problem}

First let us recall the regularization process performed in [2] :

$$
s(t, \delta)+\varepsilon s_{D}(t, \delta)=l(\delta) \quad \text { for every } \quad \delta \in H_{00}^{\frac{1}{2}}\left(\Gamma_{a}\right),
$$

which we write with our notations

$$
S(a) t+\varepsilon Q(a)^{-1} t=w_{2}+Q(a)^{-1} w_{1},
$$

or equivalently

$$
(R(a)+\varepsilon I) t=Q(a) w_{2}+w_{1} .
$$

In a classical way, the parameter $\varepsilon$ is fixed according to the noise on the measurements on $\Gamma_{0}$. It is clear that we can only estimate the modes of $t$ corresponding to the eigenvalues of $S(a)$ larger than those of the regularizing term $\varepsilon Q(a)^{-1}$. We shall define them as the computable modes

Let us define the parameter $d$ by

$$
d=\frac{2-\varepsilon-2 \sqrt{1-\varepsilon}}{\varepsilon} .
$$

Proposition 5 The computable modes for the regularization (31) are those verifying

$$
\alpha_{n} \leq \frac{1}{2 a} \ln \left(\frac{1}{d}\right)
$$

Proof

We denote by $S_{\varepsilon}$ the regularization of $S$,

$$
S_{\varepsilon}=S+\varepsilon Q(a)^{-1} .
$$

Let $\left(\lambda_{n}\right)_{n},\left(\operatorname{resp}\left(\lambda_{n}^{\varepsilon}\right)_{n}\right)$ be the eigenvalues of $S$ (resp of $S_{\varepsilon}$ ). We have

$$
\lambda_{n}^{\varepsilon}=4 \alpha_{n} \frac{e^{-2 \alpha_{n} a}}{1-e^{-4 \alpha_{n} a}}+\varepsilon \alpha_{n} \frac{1+e^{-2 \alpha_{n} a}}{1-e^{-2 \alpha_{n} a}} .
$$


The $n$th mode is computable if

$$
\lambda_{n}>\varepsilon \alpha_{n} \frac{1+e^{-2 \alpha_{n} a}}{1-e^{-2 \alpha_{n} a}}
$$

that is

$$
\frac{4 e^{-2 \alpha_{n} a}}{\left(1+e^{-2 \alpha_{n} a}\right)^{2}}>\varepsilon
$$

Setting $X=e^{-2 \alpha_{n} a}$ we must have

$$
\varepsilon X^{2}+2(\varepsilon-2) X+\varepsilon<0 .
$$

The zeroes of this polynomial are $X_{1}=d$ and $X_{2}=\frac{2-\varepsilon+2 \sqrt{1-\varepsilon}}{\varepsilon}$. So we must have

$$
\alpha_{n} \leq \frac{1}{2 a} \ln \left(\frac{1}{d}\right)
$$

Remark 3 Condition (33) shows how the number of computable modes depends on the regularization parameter $\varepsilon$ and that it decreases as a increases.

We propose now a new regularization of the optimal control problem by considering the cost function

$$
E_{\varepsilon}(\eta, \tau)=\int_{\Omega}\left(\nabla u_{1}-\nabla u_{2}\right)^{2} d x d y+2 \varepsilon \int_{\Omega} \nabla u_{1} \nabla u_{2} d x d y
$$

By calculations similar to Proposition 1 one can show that

$$
\int_{\Omega} \nabla u_{1} \nabla u_{2} d x d y=\int_{\Gamma_{0}} \Phi T d y+\int_{\Gamma_{a}}\left(Q \eta+w_{1}\right)\left(P \tau+w_{2}\right) d y .
$$

Defining

$$
A_{\varepsilon}=\left(\begin{array}{cc}
Q & -(1-\varepsilon) Q P \\
-(1-\varepsilon) P Q & P
\end{array}\right),
$$

the solution of the regularized optimal control problem is given by

$$
A_{\varepsilon}[\varphi, t]^{\prime}=(1-\varepsilon)\left[Q w_{2}, P w_{1}\right]^{\prime}
$$

which is well posed for $\varepsilon>0$ sufficiently small. After elimination, the regularized version of $R$ is

$$
R_{\varepsilon}=\left(I-(1-\varepsilon)^{2} P Q\right) .
$$

Using the former calculations in the cylindrical case its spectrum is

$$
\left\{2\left(1+(1-\varepsilon)^{2}\right) \frac{e^{-2 \alpha_{n} a}}{\left(1+e^{-2 \alpha_{n} a}\right)^{2}}+\left(2 \varepsilon-\varepsilon^{2}\right) \frac{1+e^{-4 \alpha_{n} a}}{\left(1+e^{-2 \alpha_{n} a}\right)^{2}}, \quad n \in \mathbb{N}\right\} .
$$

Setting

$$
d^{\prime}=\frac{\varepsilon+1-\sqrt{1+2 \varepsilon}}{\varepsilon},
$$

and neglecting the terms in $\varepsilon^{2}$ we have the same result as in proposition 5 :

$$
\alpha_{n} \leq \frac{1}{2 a} \ln \left(\frac{1}{d^{\prime}}\right)
$$

but as $d>d^{\prime}$, more modes could be computable by this regularization. Furthermore the influence of this regularization is less on the first modes and for small $a$ than the previous one. This is desirable as these situations correspond to less ill-posed problems. 


\section{Numerical results}

In this section we present numerical experiments on the factorization based method we propose for the data completion problem. We call this method "the direct method" because it needs the resolution of three differential equations as opposed to the method of [1] which we call iterative because it uses an iterative algorithm of minimization.

We consider problem $\left(\mathcal{P}_{0}\right)$ on $\left.\Omega=\right] 0, a[\times] 0, \pi\left[\right.$. The Cauchy data on $\Gamma_{0}$ are $\Phi=\sin (y)$ et $T=\sin (y)$. Then the harmonic function $u(x, y)=e^{x} \sin (y)$ is the solution of $\left(\mathcal{P}_{0}\right)$ and the missing data to be recovered on $\Gamma_{a}$ are $\varphi_{t h}=\frac{\partial u}{\partial n}(a, y)=e^{a} \sin (y)$ et $t_{t h}=u(a, y)=e^{a} \sin (y)$.

For the numerical experiments with the direct method, a finite difference method is used. The grid points are : $a_{i j}=(i h, j k)$ for $i=1 \ldots n$ and $j=1 \ldots n-1, n \in \mathbb{N}$, $h=\frac{a}{n}$ and $k=\frac{\pi}{n}$. There is no boundary value problem to solve. Instead we have to solve an initial value problem for the operator Riccati equations (6), (16). Let us first consider a semi-discretization with respect to the coordinate $y$ by a finite difference method. The Dirichlet to Neumann operator $P$ becomes a $(n-1) \times(n-1)$ matrix $P_{i}$

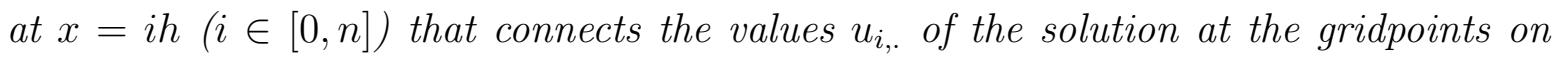
section $x=i$ th the approximation of $\frac{\partial u}{\partial x}$ on the same section. This matrix satisfies a matrix Riccati equation in $x$. After a discretization in the $x$ direction $(i \in \mathbb{N})$, this equation is solved by an explicit Euler scheme respecting stability conditions. Namely one has

$$
\left\{\begin{array}{l}
P_{i}=P_{i-1}\left(I-h P_{i-1}\right)-h \Delta_{y, k}, \\
P_{0}=0,
\end{array}\right.
$$

where $\Delta_{y, k}$ is the three diagonal matrix associated with the three points approximation to the second derivative. A similar scheme is applied to the Neumann-Dirichlet operator

$$
\left\{\begin{array}{l}
Q_{i}=Q_{i-1}\left(I-h \Delta_{y, k} Q_{i-1}\right)+h I \\
Q_{0}=0
\end{array}\right.
$$

The method has been implemented in a Scilab program.

\subsection{Comparison with the iterative method}

In this section we compare the iterative method of [1] with a regularizing term with ours. In both cases the data completion problem is set as a control problem as formulated in $1,2,3$.

For the iterative method we solve the state equation by a $P 1$ finite element method on a regular triangular meshing with the same gridpoints using the FreeFEM software. The minimization uses a conjugate gradient method.

We denote $t_{d}, t_{i}$ the Dirichlet condition on the section $\Gamma_{a}$ computed respectively by the direct method and the iterative method. Let $\operatorname{err}_{d}$ and $e r r_{i}$ be respectively the relative errors with respect to the theoretical solution $t_{t h}$ in $L^{2}$ norm of respectively $t_{d}, t_{i}$. Let time $_{1}$ be the computing time for the operators $P, Q$ and $R$, time 2 be the 
computing time for the functions $w_{1}, w_{2}$ and for solving and (19) and time $_{3}$ be the computing time for the iterative method.

For this computation we took $\varepsilon=10^{-8}$ and $a=\frac{\pi}{2}$.

\begin{tabular}{|c|c|c|c|c|c|}
\hline & time $_{1}$ (sec.) & time $_{2}$ (sec.) & err $_{d}$ & time $_{3}$ (sec.) & err $_{i}$ \\
\hline $\mathrm{n}=100$ & 2.9 & 0.135 & $10^{-2}$ & 10.53 & $9.10^{-3}$ \\
\hline $\mathrm{n}=200$ & 38.9 & 0.89 & $5.10^{-3}$ & 47.80 & $4.10^{-3}$ \\
\hline $\mathrm{n}=300$ & 198.2 & 1.88 & $3.10^{-3}$ & 124.3 & $2.10^{-3}$ \\
\hline
\end{tabular}

As we do not use the same software for the direct and iterative methods we cannot compare directly the computing times. Nevertheless Scilab being an interpreted language it is known to have slower execution times which favours the direct method for small size problems.

These results are in accordance with known facts for the comparison between direct and iterative methods for solving numerically linear systems. The complexity for factorizing a $(n, n)$ matrix is $O\left(n^{3}\right)$. In our framework the factorization is obtained by solving the Riccati equations and so this increase of complexity is in accordance with the increase of time $_{1}$. On the other hand this computation is independent of the data $(\Phi, T)$ which means that once $P, Q$ and $R$ have been computed for an extra set of data only a computation of duration time $_{2}$ is needed. As time $e_{2}$ remains small this gives an advantage to the direct method if a large set of data has to be treated.

\subsection{Comparison of regularizations}

In section 7.3 we presented a new way of regularizing the data completion problem. In this section we compare it with the one of [1]. Let err $_{d r 2}$ be the relative error between $t_{t h}$ and $t_{d}$ when the classical regularization of [1] is used.

We set $\varepsilon=10^{-2}$ and $n=50$. We study the effect of the length of the cylinder $a$.

\begin{tabular}{|c|c|c|}
\hline & err $_{d r 2}$ & err $_{d}$ \\
\hline$a=\frac{\pi}{10}$ & $10^{-2}$ & $2.10^{-3}$ \\
\hline$a=\frac{\pi}{50}$ & $10^{-2}$ & $10^{-4}$ \\
\hline$a=\frac{\pi}{100}$ & $10^{-2}$ & $4.10^{-5}$ \\
\hline
\end{tabular}

As it was stated in section 7.3 our regularization adapts itself to the case of small lengthes where the problem is less ill-posed. 


\subsection{Stability}

In this section we study the stability of the data completion problem to noise on the data. Let $e r r_{d n}$ the relative error on $t_{d}$ when a noise is added to the data. The level of the noise is $10^{-1}$. We set $n=100$ and $a=\frac{\pi}{2}$.

\begin{tabular}{|c|c|c|}
\hline & err $_{d n}$ & err $_{d}$ \\
\hline$\varepsilon=10^{-1}$ & 0.4 & 0.4 \\
\hline$\varepsilon=10^{-2}$ & $4.10^{-2}$ & $7.10^{-2}$ \\
\hline$\varepsilon=10^{-4}$ & 0.19 & $10^{-2}$ \\
\hline$\varepsilon=10^{-6}$ & 2.75 & $10^{-2}$ \\
\hline
\end{tabular}

\section{Conclusion}

The factorization method allows an explicit recovering of the boundary missing data when the Cauchy problem is rephrased into an optimal control one. In this formulation the operators $\mathrm{P}$ and $\mathrm{Q}$ do not depend on the data. The main feature of the proposed method is that if we have a collection of boundary data to recover, that is a collection of Cauchy data $(\Phi, T)$ one has only to solve the initial value problems involving $w_{1}$ and $w_{2}$. Furthermore the proposed method permits to perform analytical computations which throw new insights into the missing data recovery problem. In particular this method allowed us to propose a new way of regularizing the problem that is self adaptative for less ill-posed problems.

In our future work we will try to extend the method to more general geometries than the cylinder and to general second order self adjoint operators, and study the effect of small perturbations of the surfaces of measurements or of completion.

\section{Acknowledgments}

The authors acknowledge the financial support of INRIA associated team Enée. The LAMSIN researchers' work is supported an a regular basis by the Tunisian Ministry for Higher Education, Scientific Research and Technology.

\section{References}

[1] S. Andrieux, T.N. Baranger and A. Ben Abda. Solving Cauchy problems by minimizing an energylike functional (Institute of Physics Publishing)

[2] M. Azaiez, F. Ben Belgacem, H. El Fekih On Cauchy's problem: II. Completion, regularization and approximation Inverse Problems, 21, 6, pp 1915-1936 (2006)

[3] Bellman, R. (1957), Dynamic Programming . Princeton University Press. 
[4] Bodart O Existence of approximate controls for a semilinear equation, Inverse problems, 12, pp 27-33 (1996).

[5] N. Bouarroudj, J. Henry, B. Louro, M. Orey On a Direct Study of an Operator Riccati Equation Appearing in Boundary Value Problems Factorization, Applied mathematical sciences, 2, 46 (2008) pp 2247-2257.

[6] L. Bourgeois. A mixed formulation of quasi-reversibility to solve the Cauchy problem for Laplace's equation, Inverse Problems, 21(3), pp 1087-1104, (2005).

[7] L. Bourgeois. Convergence rates for the quasi-reversibility method to solve the Cauchy problem for Laplace's equation, Inverse Problems, 22(2), pp 413-430, (2006).

[8] L. Bourgeois, E. Lunéville. The method of quasi-reversibility to solve the Cauchy problems for elliptic partial differential equations, PAMM, 7(1), 1042101-1042102, 2007.

[9] L. Bourgeois. Conditional stability for ill-posed elliptic Cauchy problems : the case of C1,1 domains (part I), Rapport INRIA n 6585, 2008.

[10] Blum J (1989) Numerical simulation and optimal control in Plasma Physics with Application to Tokamaks, (Modern Applied Mathematics) (Chichester: Wiley / Paris: Gauthier-Villars).

[11] A. Chakib and A. Nachaoui. Convergence analysis for finite element approximation to an inverse Cauchy problem

[12] Hadamard J 1953 Lectures on Cauchy Problem in Linear Partial Equation ( New York: Dover).

[13] J. Henry, A. Ramos, Factorization of second order elliptic boundary value problems by dynamic programming Nonlinear Analysis 59 (2004) 629-647.

[14] J. Henry, A. Ramos, A Direct Study of the Riccati Equation Appearing in a Factorization Method of Second Order Elliptic Boundary Value Problems by Dynamic Programming Nonlinear Analysis (2007).

[15] M. Klibanov and F. Santosa, A computational quasi-reversibility method for Cauchy problems for Laplace's equation, SIAM j. appl. math. vol 51, No 6, pp 1653-1675 (1991)

[16] M. V. Klibanov and A. Timonov, Carleman Estimates for Coefficient Inverse Problems and Numerical Applications, VSP, Utrecht, 2004.

[17] Lattès R. and Lions J.L (1967) Méthode de Quasi-réversibilité et Applications, Dunod, Paris.

[18] J. Leblond, M. Mahjoub, J. Partington. Analytic extensions and Cauchy-type inverse problems on annular domains: stability results, $\mathrm{J}$ of Inverse Ill-Posed Problems, 2006, vol. 14, no 2, p. 189-204.

[19] Lions J.L., and Magenes E. (1968). Problèmes aux limites Non homogènes et Applications, Vol1. Dunod

[20] Lions J.L., (1968) Controle optimal de systèmes gouvernés par des équations aux dérivées partielles, Dunod

[21] T. Takeuchi and M. Yamamoto Tikhonov regularization by a reproducing kernel Hilbert space for the Cauchy problem for an elliptic equation, SIAM J. SCI. COMPUT. Vol. 31, No. 1, pp. 112-142 (2008). 